\title{
Evolution of weighted scale-free networks in empirical data
}

\author{
Y.-H. Eom, C. Jeon, and H. Jeong* \\ Department of Physics, Korea Advanced Institute of Science and Technology, 305-701 Daejon, Korea \\ B. Kahng \\ Department of Physics and Astronomy and Center for Theoretical Physics, Seoul National University, 151-747 Seoul, Korea
}

(Received 14 September 2007; published 16 May 2008)

\begin{abstract}
Weighted scale-free networks exhibit two types of degree-strength relationship: linear and nonlinear relationships between them. To understand the mechanism underlying such empirical relationships, theoretical evolution models for weighted scale-free networks have been introduced for each case. However, those models have not yet been tested with empirical data. In this study, we collect temporal records of several online bulletin board systems and a movie actor network. We measure the growth rates of degree and strength of each vertex and weight of each edge within the framework of preferential attachment (PA). We also measure the probability of creating new edges between unconnected pairs of vertices. Then, based on the measured rates, linear and nonlinear growth models are constructed. We find that indeed the dynamics of creating new edges and adding weight to existing edges in a nonlocal manner is essential to reproduce the nonlinear degreestrength relationship. We also find that the degree-driven PA rule is more appropriate to real systems rather than the strength-driven one used for the linear model.
\end{abstract}

DOI: 10.1103/PhysRevE.77.056105

PACS number(s): 89.75.-k, 89.65.-s

\section{INTRODUCTION}

The Barabási and Albert (BA) model [1], recently introduced for scale-free (SF) networks, is a distinct model compared with the random graph [2], the small-world model [3], and the static model [4], in the perspective that the network is evolving, i.e., the total number of vertices increases with time. In the BA model, the preferential attachment (PA) rule was introduced, where a new vertex is attached to existing vertices with the probability linearly proportional to the degree of the target vertices. Later, the PA rule was justified using the empirical data of the coauthorship network [5]. These pioneering studies were limited to binary networks and were later extended to weighted networks in order to model real networks. For example, the coauthorship network contains edges with weights that represent the number of papers written together between two coauthors. The dialogue network of an online bulletin board system (BBS) has edge weight, which is the frequency of dialogues between two people. The edge weight in the worldwide airport network is the number of passengers traveling between two cities. In such weighted networks, strength $s_{i}$, defined as the sum of weights on the edges connected to a given vertex $i$, is a physical quantity that is as important as the degree $k_{i}$. In general, strength and degree are related to each other in a nonlinear fashion, $s_{i} \sim k_{i}^{\theta}[6-11]$. The exponent $\theta$ can be either 1 (called the linear case) or not (called the nonlinear case), depending on the systems. The linear and nonlinear cases have been found in the scientific coauthorship network and the worldwide airport network, respectively [6].

The linear case was modeled by Barrat, Barthelemy, and Vespignani (BBV) [12]. The BBV model is generally similar

\footnotetext{
*hjeong@kaist.ac.kr
}

to the BA model, however, they are different in that the PA rule is applied to the strength instead of the degree in the BBV model. Hereafter, the former (latter) PA rule is called the strength-driven (degree-driven) PA. Once a new connection is made, the updating of the edge weight is followed locally at the edges connected to the target vertex. This local updating rule yields a linear relationship between the degree and strength. On the contrary, it was suggested that the nonlinear relationship can be achieved by the nonlocal updating of the edge weight [13]. This idea has been embodied in a few evolution models $[14,15]$. In the model proposed by $\mathrm{Bi}-$ anconi [14] (Wang et al. [15]), a target vertex is selected by the degree-driven (strength-driven) PA rule. Moreover, the edge weight is strengthened or reinforced at selected internal edges for both models. However, the dynamics of a new connection of edges between existing, but as yet unconnected vertices was not taken into account. While the two models are successful in generating a nonlinear relationship between the degree and strength, they have not yet been tested with empirical data. In this paper, we test the elements proposed in the two above-mentioned papers with empirical data. For this purpose, we measure the key quantities that drive the evolution of weighted networks from the empirical data, which are the growth rates of the degree and strength of each vertex, and the weight of each edge as a function of time, i.e., the total number of vertices. Indeed, we find that the nonlocal updating of the edge weight is an important element in obtaining the nonlinear relationship between the degree and strength. Moreover, based on the obtained results, we modify the previous model to include the case where the total number of edges increases nonlinearly with respect to the number of vertices. We find that the result obtained from the degree-driven PA rule can fit better with the empirical data, and the dynamics of adding edges between unconnected pairs of vertices must be taken into account. 
TABLE I. Summary of the statistics of the evolution of network structure for the weighted networks. $\Delta \tau$ is the temporal resolution of the data. $T$ is the period for observation. Other quantities are defined in the text.

\begin{tabular}{lcccc}
\hline \hline & Loco & Bar & Google & Movie \\
\hline Size & 7435 & 3988 & 39918 & 752682 \\
$\Delta \tau$ & 1 day & 1 day & 1 day & 1 year \\
$T$ & 1670 days & 1575 days & 1259 days & 117 years \\
$p$ & $0.51 \pm 0.01$ & $0.82 \pm 0.01$ & $0.84 \pm 0.01$ & $0.01 \pm 0.002$ \\
$q$ & $0.0068 \pm 0.0005$ & $0.026 \pm 0.001$ & $0.66 \pm 0.02$ & $0.00084 \pm 0.00028$ \\
$r$ & $0.0017 \pm 0.0001$ & $0.11 \pm 0.01$ & $0.084 \pm 0.006$ & $0.016 \pm 0.007$ \\
$\alpha_{1}$ & $1.12 \pm 0.002$ & $1.07 \pm 0.001$ & $1.04 \pm 0.002$ & $1.61 \pm 0.02$ \\
$\alpha_{2}$ & $1.85 \pm 0.008$ & $1.70 \pm 0.005$ & $0.99 \pm 0.004$ & $1.85 \pm 0.04$ \\
$\alpha_{3}$ & $2.28 \pm 0.009$ & $1.81 \pm 0.012$ & $0.84 \pm 0.01$ & $1.55 \pm 0.05$ \\
\hline \hline
\end{tabular}

\section{ANALYSIS OF EMPIRICAL DATA}

Our data set is comprised of four real networks, three BBSs (loco, bar, and Google) [16,17], and movie actor networks [18]. A BBS is an online social communication system where users post messages and others reply to them. The BBS data contain the information of user names in the from and to fields and the posting date of each message. This yields a weighted network where vertices are users and a pair of users is connected if the users exchange at least one message. The weight $w_{i j}$ of the edge between two users $i$ and $j$ is determined as the number of messages they communicate. The movie data include information on movie titles, actors, and production years. In this network, the vertices are actors and the edges between two actors $i$ and $j$ are connected if they act in the same movie. The weight $w_{i j}$ between the vertices $i$ and $j$ is the number of co-starring movies. The strength $s_{i}$ of a vertex $i$ is defined by $s_{i}=\Sigma_{j \in \operatorname{nn}(i)} w_{i j}$, where $\mathrm{nn}(i)$ is the nearest neighbor of the vertex $i$. For the BBS (loco and bar) networks, the strength distributions exhibit heavy-tailed behaviors, which were also shown in our previous works [17]. Other networks show similar behaviors. Table I shows the summary of the structural features of the weighted networks.

We examine the evolution pattern of the weighted networks in the real world, particularly focusing on the three evolution processes (Fig. 1):

(i) Addition of a new vertex: A new vertex is introduced and edges are created from the new vertex to the existing vertices. $\Pi_{\text {ext }}$ denotes the probability of connecting edge to the vertex. This process is regarded as an occurrence during a unit time interval.

(ii) Creation of internal edges: Internal edges are created between existing vertices that are unconnected. $\Pi_{\text {int }}$ is the probability of connecting the two vertices.

(iii) Reinforcement of edge weight: The weights of the selected existing edges are increased by a unit weight.

Both processes (i) and (iii) are proposed in Refs. [14,15], but (ii) is introduced here.

To be specific, the number of vertices at a given instant $\tau$ is regarded as time $t$. Let $L_{\text {ext }}(t), L_{\text {int }}(t)$, and $W_{\text {rei }}(t)$ be the numbers of edges at time $t$ created from processes (i)-(iii), respectively. Empirical measurements show that these quan- tities increase in power law as $L_{\text {ext }}(t) \approx p t^{\alpha_{1}}, L_{\text {int }}(t) \approx q t^{\alpha_{2}}$, and $W_{\text {rei }}(t) \approx r t^{\alpha_{3}}$ asymptotically. $p, q$, and $r$ are constants, and the exponents $\alpha_{1}, \alpha_{2}$, and $\alpha_{3}$ vary depending on the systems, as listed in Table I. For all networks, $\alpha_{2}$ and $\alpha_{3}$ are nontrivial, indicating that the internal activity in the form of creating internal edges and strengthening edge weights is not negligible in BBS networks. It is noteworthy that these exponents are larger than 1 for the loco, bar BBS, and movie actor networks, indicating that these systems become more densely connected with an increasing time; this is called accelerated growth. For the Google BBS network, however, the exponents $\alpha_{1}$ and $\alpha_{2}$ are close to 1 , suggesting a linear growth. The exponent $\alpha_{3}$ is less than 1 .

To understand the evolution mechanism microscopically, we measure the increasing rates of each quantity, and obtain kernels $\Pi$ that drive the dynamics for each evolution process (i)-(iii).

The kernel function $\Pi$ determines the attachment probability for topological growth and the probability of weight increase for link weight reinforcement. For the degree growth resulting from adding new vertices, the kernel function is reduced to that of preferential attachment. The function $\Pi_{\text {ext }}(k)$ is described by (a)

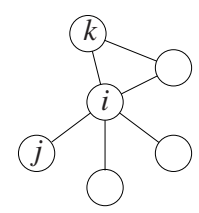

(c)

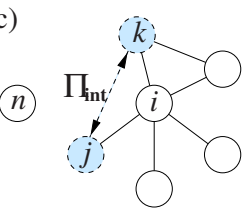

(b)

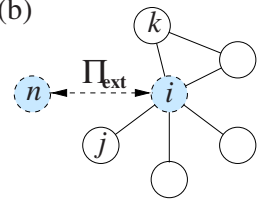

(d)

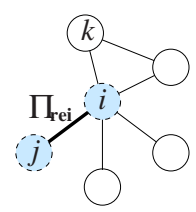

FIG. 1. (Color online) Schematic illustration of the evolution dynamics of a weighted network. (a) Initial configuration. (b) A new vertex $n$ is introduced to the system and connects to an existing vertex $i$ with probability $\Pi_{\text {ext }}$ (external growth). (c) Two existing vertices $k$ and $j$ that are still unconnected are connected with probability $\Pi_{\text {int }}$ (internal growth). (d) Edge weight between existing vertices $i$ and $j$ is increased by one with probability $\Pi_{\text {rei }}$ (edgeweight reinforcement). 
TABLE II. Numerical values of the exponents defined in the kernels.

\begin{tabular}{lcccc}
\hline \hline & Loco & Bar & Google & Movie \\
\hline$\beta_{1, k}$ & $0.94 \pm 0.08$ & $1.25 \pm 0.13$ & $1.36 \pm 0.14$ & $0.64 \pm 0.06$ \\
$\beta_{1, s}$ & $0.64 \pm 0.05$ & $0.77 \pm 0.09$ & $1.14 \pm 0.09$ & $0.62 \pm 0.06$ \\
$\beta_{2, k}$ & $0.83 \pm 0.08$ & $0.67 \pm 0.1$ & $0.70 \pm 0.08$ & $0.50 \pm 0.03$ \\
$\beta_{2, s}$ & $0.41 \pm 0.02$ & $0.27 \pm 0.04$ & $0.62 \pm 0.08$ & $0.48 \pm 0.03$ \\
$\beta_{3, w}$ & $0.87 \pm 0.08$ & $0.87 \pm 0.09$ & $1.40 \pm 0.07$ & $1.01 \pm 0.08$ \\
\hline \hline
\end{tabular}

$$
\Pi_{\mathrm{ext}}(k, t)=\frac{\sum_{i} \Delta k_{i}}{n(k, t)} \approx C(t) k^{\beta},
$$

where $\Delta k_{i}$ is the number of new links that attach to vertex $i$ with degree $k$ during $\Delta t$, and $n(k, t)$ is the number of vertices with degree $k$ at time $t$. Because of the data fluctuation, we use a time-average kernel function. That is, $\Pi_{\text {ext }}$ $=\left\langle\Pi_{\text {ext }}(k, t)\right\rangle$. The increasing rate of degree $k_{i}$ of a vertex $i$ by the attachment of a new edge from a new vertex is measured as follows:

$$
\frac{d k_{i}}{d t}=m_{1} \Pi_{\mathrm{ext}, k}\left(k_{i}\right)
$$

where $m_{1}=\left\langle d L_{\text {ext }} / d t\right\rangle$ is the average number of edges attached from a new vertex during a unit time interval. $\langle\cdots\rangle$ is the average over different times. It was found that $\Pi_{\text {ext, } k}\left(k_{i}\right)$ $\sim k_{i}^{\beta_{1, k}}$ with $\beta_{1, k} \approx 0.94$ for the loco BBS network. The increasing rate of degree $k_{i}$ can also be measured as a function of strength,

$$
\frac{d k_{i}}{d t}=m_{1} \Pi_{\mathrm{ext}, s}\left(s_{i}\right)
$$

It was found that $\Pi_{\mathrm{ext}, s}\left(s_{i}\right) \sim s_{i}^{\beta_{1, s}}$ with $\beta_{1, s} \approx 0.64$ for the loco BBS network. The measured values of the exponents $\beta_{1, k}$ and $\beta_{1, s}$ for other systems are listed in Table II.

The increasing rate of quantity $k_{i j}$, which is the average number of links newly connected between unconnected vertices $i$ and $j$, is measured as a function of $k_{i} k_{j}$,

$$
\frac{d k_{i j}}{d t}=m_{2} \Pi_{\text {int }, k}\left(k_{i} k_{j}\right),
$$

and measured as a function of $s_{i} s_{j}$,

$$
\frac{d k_{i j}}{d t}=m_{2} \Pi_{\text {int }, s}\left(s_{i} s_{j}\right),
$$

where $m_{2}=\left\langle d L_{\text {int }} / d t\right\rangle$ is the average number of internal edges attached between unconnected vertices during a unit time interval. It was found that $\Pi_{\text {int }, k}\left(k_{i} k_{j}\right) \sim\left(k_{i} k_{j}\right)^{\beta_{2, k}}$ with $\beta_{2, k}$ $\approx 0.83$ and $\Pi_{\text {int }, s}\left(s_{i} s_{j}\right) \sim\left(s_{i} s_{j}\right)^{\beta_{2, s}}$ with $\beta_{2, s} \approx 0.41$ for the loco BBS network. The measured values of the exponents $\beta_{2, k}$ and $\beta_{2, s}$ for other systems are listed in Table II.

The increasing rate of edge weight $w_{i j}$ is
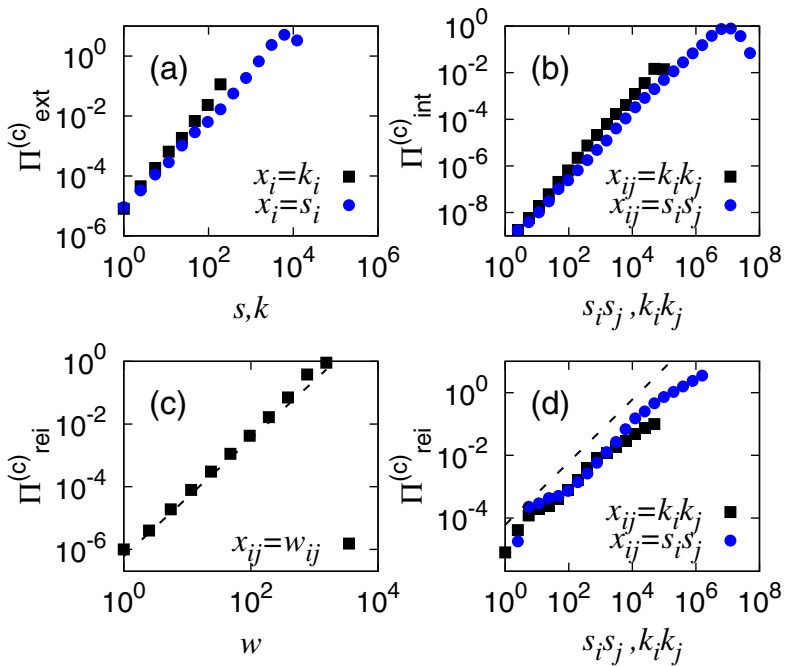

FIG. 2. (Color online) Cumulative functions of the kernels defined in Eqs. (2)-(4) as functions of their argument for the loco BBS network. (a) $\Pi_{\text {ext }}$ for the process (i). (b) $\Pi_{\text {int }}$ for the process (ii). (c) $\Pi_{\text {rei }}$ as a function of weight $w_{i j}$ for the process (iii). The straight lines are guidelines with slopes of 1.87 (d) $\Pi_{\text {rei }}$ as functions of $k_{i} k_{j}$ or $s_{i} s_{j}$. The dashed lines are guides to the eye showing slope 1 .

$$
\frac{d w_{i j}}{d t}=m_{3} \Pi_{\text {rei }}\left(w_{i j}\right)
$$

where $m_{3}=\left\langle d W_{\text {rei }} / d t\right\rangle$ is the average number of reinforced weights during a unit time interval. It was found that $\Pi_{\text {rei }}\left(w_{i j}\right) \sim w_{i j}^{\beta_{3, w}}$ with $\beta_{3, w} \approx 0.87$ for the loco BBS network. The measured values of the exponents $\beta_{3, w}$ for other systems are listed in Table II. Notice that to calculate $\Pi_{\text {ext, } k}\left(k_{i}\right)$, we calculate the increment of the degree $\Delta k_{i}^{\text {ext }}(\tau)$ of each vertex $i$ at a given instant $\tau$ using only the connection from an introduced vertex. If the total number of edges generated from the new vertex at a given instant $\tau$ is $\Delta L_{\text {ext }}(\tau)$, then $\Pi_{\text {ext }, k}\left(k_{i}, \tau\right)=\Delta k_{i}^{\text {ext }}(\tau) / \Delta L_{\text {ext }}(\tau)$. Since we are interested in the degree dependence of the kernel $\Pi_{\text {ext, } k}\left(k_{i}\right)$, we take the average over time and vertex indices with the same degree. To reduce the noise level, we use the cumulative function defined as $\Pi^{(c)}(x)=-\int_{\infty}^{x} \Pi(y) d y$, as shown in Fig. 2. The other kernels $\Pi_{\text {ext }}, \Pi_{\text {int }}$, and $\Pi_{\text {rei }}$ are determined in the same manner and shown in Figs. 2(a)-2(c), respectively. We found that $\Pi_{\text {rei }}\left(w_{i j}\right)$ is almost independent of $k_{i} k_{j}$ and $s_{i} s_{j}$ in Fig. 2(d) since it follows $\Pi_{\text {rei }}^{(c)}(x) \sim x$, which corresponds to $\Pi_{\text {rei }}(x)$ $=$ const. The numerical results suggest that a relationship between the degree and strength exists in the form of $s \sim k^{\theta}$ with $\theta \approx 1.4$ for the loco BBS network. Thus, $\beta_{1, k} \simeq \theta \beta_{1, s}$ and $\beta_{2, k} \simeq \theta^{2} \beta_{2, s}$.

\section{LINEAR GROWTH MODEL}

Based on the numerical data, we construct a linear growth model for the weighted network, where the total numbers of vertices and edges linearly increase with time. This case can be seen in the Google BBS network. The dynamic rule for the model is as follows: (i) At each time step, a new vertex is introduced into the system and it attaches $\ell_{1}$ edges to the 
existing vertices selected by the linear PA rule applying to the degree. The target vertices are selected with the probability linearly proportional to their degrees. Here, we use the degree-driven PA rule only to solve the problem analytically. A minimal weight $w_{0}=1$ is assigned to each new edge. (ii) At the same time, $\ell_{2}$ edges are created between those numbers of unconnected pairs of existing vertices $i$ and $j$, selected with a probability linearly proportional to the product of their degrees, i.e., $k_{i} k_{j}$. (iii) $\ell_{3}$ existing edges are selected and their weights are increased by $w_{0}$. The selection is made following the linear PA rule applied to the weight $w_{i j}$. The above rules are repeated until there are $N$ vertices in the system. This linear growth model enables us to understand the dynamic process analytically. [19]

The dynamic equation for processes (i) and (ii) is given as

$$
\frac{\partial k_{i}}{\partial t}=\left(\ell_{1}+2 \ell_{2}\right) \frac{k_{i}}{\sum_{j} k_{j}},
$$

where $\Sigma_{j} k_{j}$ is given by $2\left(\ell_{1}+\ell_{2}\right) t$. The solution is given as

$$
k_{i}(t)=\ell_{1}\left(t / t_{i}\right)^{\eta_{1}},
$$

where $\eta_{1}=\left(\ell_{1}+2 \ell_{2}\right) / 2\left(\ell_{1}+\ell_{2}\right)$ and $t_{i}$ is the time at which vertex $i$ was introduced into the system.

The dynamic equation for process (iii) is

$$
\frac{\partial w_{i j}}{\partial t}=2 \ell_{3} w_{0} \frac{w_{i j}}{\sum_{m, m^{\prime}} w_{m, m^{\prime}}} .
$$

Then, the solution is

$$
w_{i j}=w_{0}\left(t / t_{i, j}\right)^{\eta_{2}},
$$

where the exponent $\eta_{2}=\ell_{3} /\left(\ell_{1}+\ell_{2}+\ell_{3}\right)$ and $t_{i, j}$ is the time at which the edge $(i, j)$ is created.

Combining Eqs. (7) and (10), the dynamic equation for the strength evolution is written as

$$
\begin{aligned}
\frac{\partial s_{i}}{\partial t} & =\sum_{j \in \mathrm{nn}(i)} \frac{\partial w_{i j}}{\partial t}+\frac{\partial k_{i}}{\partial t} \\
& =2 \ell_{3} w_{0} \sum_{j \in \mathrm{nn}(i)} \frac{w_{i j}}{\sum w_{m, m^{\prime}}}+\frac{\partial k_{i}}{\partial t} \\
& =\frac{\ell_{3}}{\left(\ell_{1}+\ell_{2}+\ell_{3}\right) t} s_{i}+\frac{\ell_{1}+2 \ell_{2}}{2\left(\ell_{1}+\ell_{2}\right) t} \ell_{1}\left(t / t_{i}\right)^{\eta_{1}} .
\end{aligned}
$$

We find that in a long time limit

$$
s_{i} \propto \begin{cases}k_{i} & \text { if } \eta_{1}>\eta_{2}, \\ k_{i} \ln k_{i} & \text { if } \eta_{1}=\eta_{2}, \\ k_{i}^{\eta_{2} / \eta_{1}} & \text { if } \eta_{1}<\eta_{2} .\end{cases}
$$
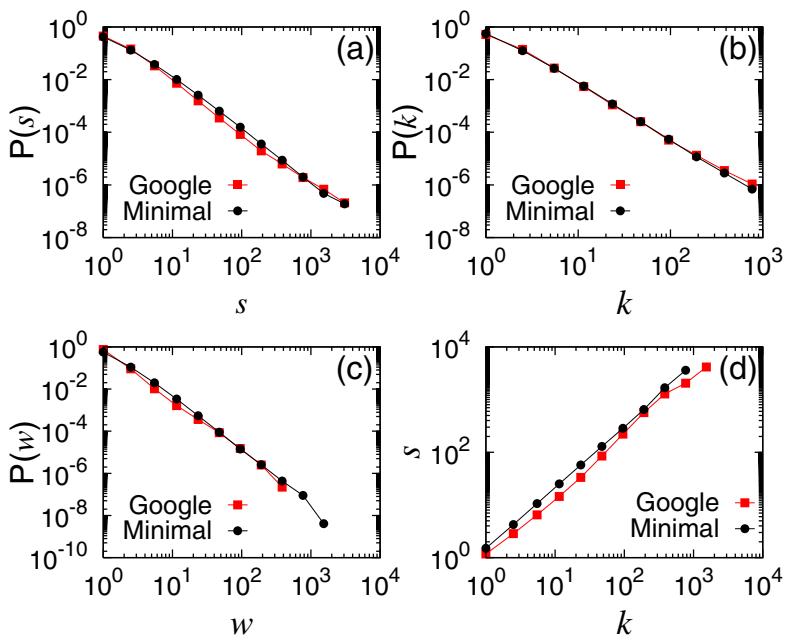

FIG. 3. (Color online) The distributions of several physical quantities — strength (a), degree (b), weight (c) for the Google BBS (red square with solid line) — and the comparison with those obtained from the linear model (black square with solid line). The degree-strength relationship is also compared (d). The numerical data are averaged over 30 network configurations with the same size as $N=39918$ of the Google BBS.

Consequently, the model generates a degree-strength relationship that is linear when $\eta_{1}>\eta_{2}$, but nonlinear when $\eta_{1}$ $<\eta_{2}$. The above result is reduced to that of the model introduced by Bianconi [14] where step (ii), the creation of new internal links, is ignored, i.e., $\ell_{2}=0$. Further, the selection rule for step (iii) is slightly different. In Ref. [14], once a vertex is chosen with the probability proportional to its strength $\Pi_{i}=s_{i} / \Sigma_{j} s_{j}$, then its partner for connection is chosen with a probability proportional to its weight $\Pi_{i j}=w_{i j} / \Sigma_{\ell} w_{i \ell}$, where the index $\ell$ is the nearest neighbor of $i$. However, in the proposed model, we choose target edges with the probability given by Eq. (9). However, the two methods reduce to the same result.

Next, using the relation $\gamma=1+1 / \eta$ previously derived in Ref. [20] for the linear kernel case, we obtain the exponents associated with the power-law decaying behaviors of the degree and edge-weight distributions. They are obtained as $\gamma_{k}$ $=2+\ell_{1} /\left(\ell_{1}+2 \ell_{2}\right)$ and $\gamma_{w}=2+\left(\ell_{1}+\ell_{2}\right) / \ell_{3}$, respectively. Then the exponent associated with the power-law decaying behavior of the strength distribution is obtained using Eq. (14) as $\gamma_{s}=\gamma_{k}$ when $\eta_{1}>\eta_{2}$ and $\gamma_{s}=\gamma_{w}$ when $\eta_{1}<\eta_{2}$.

We compare the analytic results with the empirical data obtained from the Google BBS network, of which $\alpha_{1} \approx \alpha_{2}$ $\approx 1$. Even though the kernels of $\Pi_{\text {ext }}$ and $\Pi_{\text {int }}$ for the Google BBS depend nonlinearly on the degree and the product of the degrees, the analytic solutions fit well with the empirical results, as shown in Fig. 3.

\section{NONLINEAR GROWTH MODEL}

Here, we study the nonlinear evolution case where the total number of edges increases nonlinearly as a function of the total number of vertices. We use the degree-driven and strength-driven PA rules, with the respective measured val- 
ues of $\left(\beta_{1, k}, \beta_{2, k}\right)$ and $\left(\beta_{1, s}, \beta_{2, s}\right)$ of the loco BBS network in Table II, and compare the respective results with the empirical data.

The growth of the network starts from an initial configuration composed of $N_{0}=10$ vertices, which are sparsely connected, for example, forming a ring, allowing for internal edge connections at a later stage. Otherwise, the internal edge growth becomes trivial. Each edge has a preassigned weight $w_{0}=1$. The network evolves under three evolution dynamics: (i) the addition of a new vertex and its connection to the existing vertices, (ii) the creation of internal edges, and (iii) the reinforcement of the weights of existing edges. For steps (i) and (ii), degree-driven and strength-driven PA rules are applied, respectively. Thus, two types of networks result. To be specific, the construct rules are as follows:

(i) At each time step, a new vertex is introduced in the system. Depending on time step $t$, the number of edges emanating from the new vertex varies as $\ell_{1}(t)=p t^{\alpha_{1}}-p(t-1)^{\alpha_{1}} \cdot p$ and $\alpha_{1}$ are chosen from Table I in order to compare the resulting network with the empirical data. The target vertex $i$ for each edge is chosen with the probability,

$$
\Pi_{\mathrm{ext}}\left(x_{1, i}\right)=\frac{x_{1, i}^{\beta_{1}}}{\sum_{j} x_{1, j}^{\beta_{1}}},
$$

where $x_{1, i}$ can be $k_{i}\left(s_{i}\right)$ for the degree-driven (strengthdriven) case. Then, $\beta_{1}$ is given as $\beta_{1, k}$ and $\beta_{1, s}$ in Table II, respectively. The initial weight of each new edge is given as $w_{0}=1$. This process reflects the dialogues between the new user and the existing users.

(ii) $\ell_{2}(t)=q t^{\alpha_{2}}-q(t-1)^{\alpha_{2}}$ edges are created between unconnected pairs of vertices, where $q$ and $\alpha_{2}$ are given in Table I. The pair of target vertices for each edge is chosen with the probability

$$
\Pi_{\mathrm{int}}\left(x_{2, i j}\right)=\frac{x_{2, i j}^{\beta_{2}}}{\sum_{i, j} x_{2, i j}^{\beta_{2}}},
$$

where $x_{2, i j}$ is $k_{i} k_{j}\left(s_{i} s_{j}\right)$ for the degree-driven (strengthdriven) case. Correspondingly, $\beta_{2}$ is $\beta_{2, k}$ and $\beta_{2, s}$ adopted from Table II, respectively. The addition of multiple edges to the same pair of vertices is not allowed. The weight of each new edge is also given as $w_{0}=1$. This process reflects the dialogues between the existing users who have not exchanged any messages previously.

(iii) $\ell_{3}(t)=r t^{\alpha_{3}}-r(t-1)^{\alpha_{3}}$ edges are chosen from all connected pairs of vertices with the probability given below, and their weights are strengthened by $w_{0}=1 . r$ and $\alpha_{3}$ are given in Table I. The probability is given as

$$
\Pi_{\mathrm{rei}}\left(w_{i j}\right)=\frac{w_{i j}^{\beta_{3}}}{\sum_{i, j} w_{i j}^{\beta_{3}}},
$$

where $\beta_{3}$ is given in Table II. Multiple additions of edge weights to the same pair are allowed in this process. This process reflects the additional message exchange between users who have exchanged messages previously.
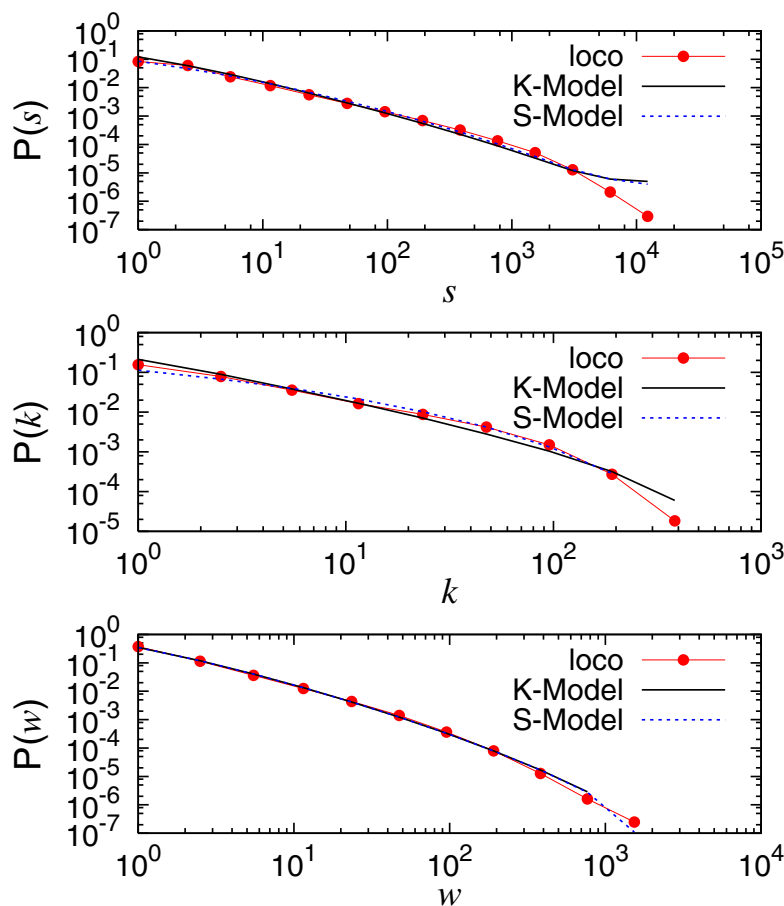

FIG. 4. (Color online) The distribution of several quantitiesstrength (a), degree (b), and weight (c) for the loco BBS network. The empirical data (red square with solid line) are compared with the results obtained from the models with the degree-driven (solid line) and the strength-driven (dotted line) PA rule. The simulation data are averaged over 30 network configurations of the same size of the loco $N=7435$.

This nonlinear model is reduced to the linear model when $\alpha_{1}=\alpha_{2}=\alpha_{3}=1$ and $\beta_{1}=\beta_{2}=\beta_{3}=1$ for the degree-driven case only. Numerical simulations were performed with the measured parameters listed in Tables I and II for the loco BBS network and the other networks. The results of the degree, strength, and edge weight distribution for the model network are shown in Fig. 4, where the degree-driven (strengthdriven) case is denoted by a solid line (dotted line). The strength and edge-weight distributions obtained from the simulations are in good agreement with the empirical data for the loco BBS network, but the degree distributions obtained from the model based on both the degree-driven and strength-driven PA slightly deviate from the corresponding empirical one.

We examined the degree-strength relationship $s \sim k^{\theta}$ [6-11] for the model network in Fig. 5(a) and compared it with the empirical data obtained from the loco BBS network. The two versions of the model network exhibit different values of $\theta$. The degree-based model, i.e., with the degreedriven PA rule, produces a closer value $\theta_{k} \approx 1.56$ to the value $\theta_{\text {loco }} \approx 1.40$ obtained from the loco BBS network than the value $\theta_{s} \approx 1.81$ obtained from the strength-based model. In addition, we calculated the confidence interval using the bootstrap procedure to compare the measured values of $\theta$ from the empirical data, the degree based model, and strength based model. The 95\% confidence interval of $\theta$ for the loco BBS data runs from 1.36 to 1.43 . Those obtained from the degree-based and the strength-based models run 

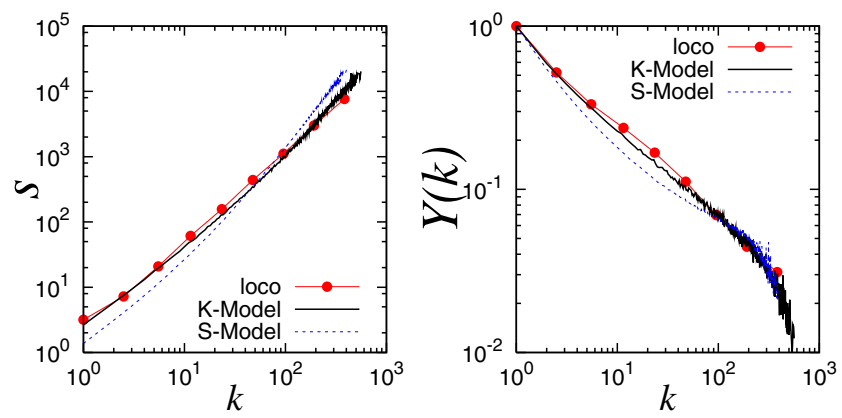

FIG. 5. (Color online) The degree-strength relationship (a). The data are obtained from the empirical data (circle), the degree-driven model (solid line), and the strength-driven model (dotted line). The weight heterogeneity as a function of degree (b). The data are obtained from the empirical data (circle), the degree-driven model (solid line), and the strength-driven model (dotted line). The simulation data are averaged over 30 network configurations of the same size as that of the loco $N=7435$.

from 1.52 to 1.59 , and from 1.76 to 1.86 , respectively. This result supports our conclusion that the degree-based model fit better than the strength-based model to reproduce the empirical result. We also examined the heterogeneity of the weights on the edges connected to a given vertex introduced in Refs. [21-23]. We measured $Y_{i}^{2}=\Sigma_{j}\left(w_{i j} / s_{i}\right)^{2} \sim k_{i}^{-\nu}$ [21]. If the weights are homogeneous, then it would be $Y_{i}^{2} \sim 1 / k_{i}$. If a dominant edge with weight $w_{i, j} \sim s_{i}$ exists, then $Y_{i}^{2} \sim O(1)$. As shown in Fig. 5(b), the empirical data of the weight heterogeneity for the loco network is more closely reproduced by the degree-based model than by the strength-based model as $\nu_{\text {loco }} \approx 0.66, \nu_{k} \approx 0.66$, and $\nu_{s} \approx 0.50$. We obtained a $95 \%$ confidence interval of $\nu$ for the loco BBS data that runs from 0.63 to 0.70 from the empirical loco data using the bootstrap procedure. This range is close to that $[0.65,0.68]$ obtained from the degree-based model, but deviates from that $[0.48,0.52]$ obtained using the strength-based model. The results for other BBS networks showed similar behaviors. Therefore, we conclude that the degree-based model is better than the strength-based model for reproducing the structural features of BBS networks.

\section{CONCLUSION}

We have analyzed the evolution records of real weighted BBS networks to understand the growth mechanism of weighted networks. We measured the growth rates of the degree and strength of each vertex and the weight of each edge as a function of time, that is, the total number of vertices. Based on the measured results, we constructed two evolving weighted network models. The models had three common elements: (i) The addition of new vertices, (ii) the addition of new internal edges between two previously unconnected vertices, and (iii) the strengthening of weights on existing edges. Processes (ii) and (iii) were applied independently of (i), so that the dynamics arising on the edges occurred in a nonlocal manner. In processes (i) and (ii), the degree-driven PA rule performs better than the strengthdriven PA rule in reproducing the features of real systems as a rule of choosing target vertices. Depending on the ratio between the growth rates of the numbers of vertices and edges, we established linear and nonlinear growth models. The Google BBS network can be reproduced using the linear growth model and the other two BBS networks and movie actor network can be reproduced through the nonlinear growth models. Our study is meaningful from the perspective that the existing concepts and models for weighted networks are tested with empirical data. We find that the existing models are overall successful in reproducing the empirical results; however, the nonlinear growth model must be used to match the empirical results.

\section{ACKNOWLEDGMENTS}

This work was supported by Acceleration Research CNRC of MOST/KOSEF (H.J., B.K.) and Grant No. R012005-000-1112-0 (Y.-H.E., C.J.) from Korea Science and Engineering Foundation (KOSEF).
[1] A.-L. Barabási and R. Albert, Science 286, 509 (1999).

[2] P. Erdös and A. Rényi, Publ. Math. Inst. Hung., Acad Sci. 5, 17 (1960).

[3] D. J. Watts and S. H. Strogatz, Nature (London) 393, 440 (1998).

[4] K.-I. Goh, B. Kahng, and D. Kim, Phys. Rev. Lett. 87, 278701 (2001).

[5] H. Jeong, Z. Neda, and A.-L. Barabasi, Europhys. Lett. 61, 567 (2003).

[6] A. Barrat, M. Bathelemy, R. Pastor-Satorras, and A. Vespignani, Proc. Natl. Acad. Sci. U.S.A. 101, 3747 (2004).

[7] J.-P. Onnela, J. Saramaki, J. Hyvonen, G. Szab, M. de Menezes, K. Kaski, A.-L. Barabási, and J. Kertész, New J. Phys. 9, 179 (2007).

[8] A. de Montis, M. Barthelemy, A. Chessa, and A. Vespignani, Environ. Plan. B: Plan. Des. 34, 905 (2007).
[9] S. Valverde, G. Theraulaz, J. Gautrais, V. Fourcassie, and R. V. Sole, e-print arXiv:physics/0602003, IEEE Intell. Syst.

[10] S. Valverde and R. V. Sole, Phys. Rev. E 76, 046118 (2007).

[11] Q. Ou, Y.-D. Jin, T. Zhou, B.-H. Wang, and B.-Q. Yin, Phys. Rev. E 75, 021102 (2007).

[12] A. Barrat, M. Barthelemy, and A. Vespignani, Phys. Rev. Lett. 92, 228701 (2004)

[13] K.-I. Goh, B. Kahng, and D. Kim, Phys. Rev. E 72, 017103 (2005).

[14] G. Bianconi, Europhys. Lett. 71, 1029 (2005).

[15] W.-X. Wang, B.-H. Wang, B. Hu, G. Yan, and Q. Ou, Phys. Rev. Lett. 94, 188702 (2005).

[16] loco.kaist.ac, kr: bar.kaist.ac.kr: http://bbs.keyhole.com

[17] K.-I. Goh, Y.-H. Eom, H. Jeong, B. Kahng, and D. Kim, Phys. Rev. E 73, 066123 (2006).

[18] http://www.imdb.com 
[19] S. N. Dorogovtsev and J. F. F. Mendes, Europhys. Lett. 52, 33 (2000).

[20] S. N. Dorogovtsev, J. F. F. Mendes, and A. N. Samukhin, Phys. Rev. Lett. 85, 4633 (2000).

[21] M. Barthelemy, A. Barrat, R. Pastor-Satorras, and A. Vespig- nani, Physica A 346, 34 (2005).

[22] E. Almaas, B. Kovacs, T. Vicsek, Z. N. Oltvai, and A.-L. Barabasi, Nature (London) 427, 839 (2004).

[23] S. H. Lee, P. J. Kim, and H. Jeong (unpublished). 